\title{
Review \\ The role of biomarkers in community-acquired pneumonia: predicting mortality and response to adjunctive therapy
}

\author{
Jean-Paul Mira ${ }^{1}$, Adeline Max ${ }^{1}$ and Pierre-Regis Burgel ${ }^{2}$
}

\begin{abstract}
${ }^{1}$ Medical Intensive Care Unit, Cochin University Hospital, Assistance Publique, Hôpitaux de Paris, Université Paris Descartes, and INSERM U567, Paris 75014, France

${ }^{2}$ Pneumology Department, University Hospital, Assistance Publique, Hôpitaux de Paris, Université Paris Descartes, Paris 75014, France
\end{abstract}

Corresponding author: Jean-Paul Mira, jean-paul.mira@cch.aphp.fr

Published: 26 November 2008

This article is online at http://ccforum.com/content/12/S6/S5

(c) 2008 BioMed Central Ltd
Critical Care 2008, 12(Suppl 6):S5 (doi:10.1186/cc7028)

Although CAP is very common, it remains a common cause of death. Hence, severe CAP has been reported to be the largest single cause of mortality from infectious diseases in industrialized countries [4]. For example, a study conducted in the USA compared outcomes in more than 150,000 patients aged over 65 years who were hospitalized for CAP with those in 800,000 control individuals matched for age, sex and race who were admitted to hospital for conditions other than pneumonia [4]. The in-hospital mortality rate among CAP patients was significantly higher, at $11 \%$, than that in non-CAP patients $(5.5 \% ; P<0.001)$. Surprisingly, the 1 -year mortality rate among CAP patients remained significantly higher than that in control individuals $(41 \%$ versus $29 \%$; $P<0.001)$. Thus, mortality rates in patients with severe CAP remain high and are discussed in detail elsewhere in this supplement [5]. The factors that underlie the poor short-term and long-term survival rates in patients with CAP and severe CAP are not yet completely understood. However, aspects of the pathophysiology of the disease, reflected in some of the recently described biomarkers and genomic markers, may contribute to increased understanding.

\section{Introduction}

Community-acquired pneumonia (CAP) accounts for 1.3 million hospitalizations each year in the USA, at an annual cost of $\$ 8.4$ billion [1]. The average duration of hospitalization for CAP managed on the ward is 6 days, at a cost of $\$ 7,500$. If intensive care unit (ICU) admission (for severe $\mathrm{CAP})$ is required, then the stay increases to 23 days, at a cost of $\$ 21,144$. The average cost for managing pneumonia in the UK was estimated at $£ 100$ per episode, as compared with $£ 1,700$ to $£ 5,100$ for hospitalized (severe CAP) patients. Hospitalization accounted for $87 \%$ of the total annual cost $[2,3]$.
Hence, in addition to being an infectious disease, severe CAP elicits major systemic responses that could also allow this pathology to be classified as inflammatory (indicated by systemic release of large amounts of proinflammatory cytokines and lipid mediators) and as a disease of haemostasis disorders (demonstrated by increased coagulation) [4]. As such, this review focuses primarily on biomarkers that may indicate susceptibility to CAP or severity of the two systemic responses (inflammation and coagulation). It also discusses the potential for genetic markers to indicate increased individual patient susceptibility to CAP.

$A U C=$ area under the receiver operating curve; $C A P=$ community-acquired pneumonia; $C R P=C$-reactive protein; CURB- $65=\mathrm{Confusion}, \mathrm{Urea}$, Respiratory Rate, Blood pressure and age over 65 years; HR = hazard ratio; ICU = intensive care unit; IL = interleukin; PCT = procalcitonin; proADM = pro-adrenomedullin; proANP = pro-atrial natriuretic peptide; proVAP = pro-vasopressin; PSI = Pneumonia Severity Index; SNP = single nucleotide polymorphism; TNF $=$ tumour necrosis factor. 
Any review of biomarkers should keep in mind the intended purpose of the marker and the setting in which it is to be used. Among potential uses of biomarkers in clinical research, the following can be distinguished: to identify patients suitable for enrolment in a trial based on the nature and severity of their disease; to elucidate a drug's mechanism of action; to demonstrate that a drug is having a particular biological effect; to establish which groups of patients within a trial population exhibit the greatest beneficial response or detrimental response; and, possibly, as a surrogate for outcome.

In the specific clinical setting of patients suspected of having CAP, we can envisage using biomarkers as an aid to identify the presence or absence of infection; to establish disease severity, risk for progression and death, and hence the need for hospitalization or admission to the ICU; to select those patients who are most likely to benefit from a specific drug or other intervention, especially those at high risk for mortality; and to monitor the efficacy of therapy and determine the need for escalation or de-escalation of treatment.

\section{Clinical stratification of risk}

Several risk stratification schemes for CAP patients have been developed and validated [5]. These include the Pneumonia Severity Index (PSI), which is based on 20 variables; the CURB-65 scale (incorporating Confusion, Urea, Respiratory Rate, Blood pressure, and age over 65 years; the simpler CRB-65 scale; and most recently the combined Infectious Diseases Society of America/American Thoracic Society guidelines for the management of CAP, which rely on the identification of major and minor clinical and laboratory criteria to determine whether patients should be admitted to the ICU [6].

A recent retrospective study of specificity and sensitivity in 419 patients with CAP [7] showed that the CURB-65 score outperformed the Standardized Early Warning Score and the systemic inflammatory response syndrome criteria as a predictor of mortality. The authors concluded that CURB-65 may be preferred over systemic inflammatory response syndrome criteria and Standardized Early Warning Score in the initial prognostic assessment of patients with CAP.

A larger prospective study conducted in 1,016 patients with CAP [8] investigated the ability of CURB-65, CRB-65 and PSI to predict 30-day mortality. The area under the receiver operating curve (AUC) for CURB-65 (0.733) was very similar to that for PSI (0.736) and slightly higher than that for CRB65 (0.694). All three systems had high negative predictive values but relatively low positive predictive values. CURB-65 can be recommended because of its simplicity and ability to identify patients at low risk.

As a generalization, these clinic-based systems, although relatively good at quickly selecting low-risk patients who can be managed as outpatients, have not been particularly effective at identifying patients at highest risk who are most likely to benefit from entry into trials of novel therapies. As such, physicians still lack effective means to establish disease severity, likelihood of deterioration, requirement for ICU admission and suitability for specific treatments. It is within this context that the search for reliable biomarkers assumes importance.

\section{Biomarkers as prognostic factors Procalcitonin and $\mathbf{C}$-reactive protein}

Muller and coworkers [9] recently reported a study including 545 patients with radiographic findings suggestive of CAP who were admitted to the emergency department. Subsequently, 373 patients were confirmed as having CAP and were included for determination of the best biological diagnosis and prognostic biomarker in this context. Procalcitonin (PCT) was clearly more accurate in differentiating CAP from other conditions than was C-reactive protein (CRP) or total leucocyte count. The AUCs for the three markers were $0.88,0.73$ and 0.69 , respectively. In contrast, all clinical signs that are routinely used to diagnose CAP, such as fever, sputum and chest auscultation, appeared to be very poor indicators of CAP (AUC between 0.3 and 0.6). In this study, PCT was also a better predictor of bacteraemia than the other markers. Moreover, there was a significant relationship between PSI category and PCT levels. In contrast, neither CRP nor leucocyte count exhibited any correlation with the PSI category.

Among patients in the highest PSI category (class V), the mean PCT was markedly elevated. Unexpectedly, however, many patients within this high-risk group had a normal level of PCT. Data from the University of Pittsburgh Genetic Inflammatory Mediator Study (GenIMS) [10], which is one of several recent attempts to gather information on large cohorts of CAP patients, provide further information on this group of patients. More than 2,000 patients with clinically and radiographically diagnosed CAP were enrolled in this study. Among them, 1,651 (who became the study cohort) had their PCT level measured on day 1. Even among patients classified as PSI group IV or V, those whose PCT levels were low at the outset $(<0.1 \mathrm{ng} / \mathrm{ml})$ had negligible 30-day and 90-day mortality rates (Figure 1). They also had a relatively short length of hospital stay and little likelihood of requiring admission to the ICU. This was also true among patients who fell into group 3 according to the CURB-65 system. Normal PCT is therefore an indicator of low risk even when scores based on clinical factors are high. Incorporation of the PCT biomarker would enhance the prognostic value of these systems of risk stratification.

\section{Other prohormones}

A multicentre, prospective study conducted by the German CAPNETZ group investigated the ability of several prohormones to predict disease severity and risk for death in 
Figure 1

\section{PSI Class IV}

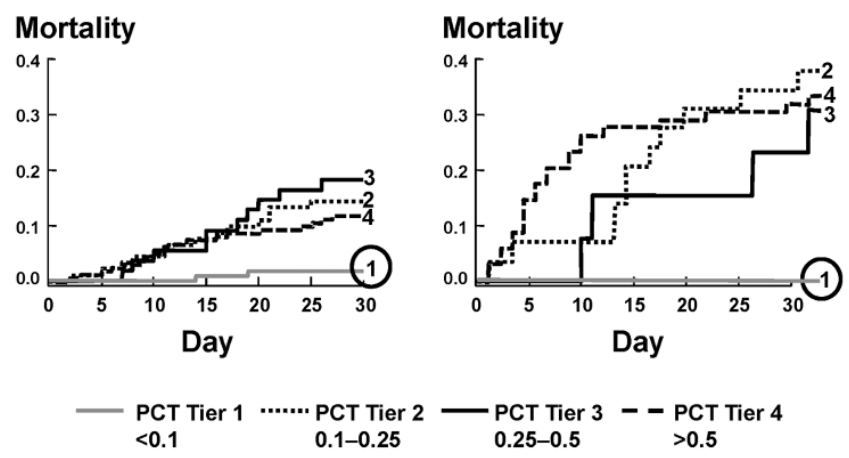

PCT level and PSI prognostic value. Low levels $(<0.1 \mathrm{mg} / \mathrm{ml})$ of PCT at baseline are predictive of survival, even in PSI group IV and V patients, although the distinction is less defined in PSI group V patients. Reproduced with permission from Huang and coworkers [10]. PCT, procalcitonin; PSI, Pneumonia Severity Index.

589 patients with proven CAP [11]. As previously reported, PCT was of significant but modest prognostic value. In accordance with the data from the Pittsburgh study described above [10], 30-day survival was more than 98\% among patients with a PCT level under $0.15 \mathrm{ng} / \mathrm{ml}$. Pro-atrial natriuretic peptide (proANP), using a cut off of $116 \mathrm{pmol} / \mathrm{l}$, had a similar prognostic significance for mortality. The most predictive factor was pro-vasopressin (proAVP); $98 \%$ of patients with a proAVP value of $28.8 \mathrm{pmol} / \mathrm{l}$ or less survived, as compared with $83 \%$ of patients with higher values (Figure 2).

Although CRP exhibited no relationship with CRB-65 score, levels of PCT, proANP and proAVP increased with disease severity [11]. Patients who died within 28 days had median proANP and proAVP levels at baseline that were significantly higher than those in patients who survived. In multivariate analysis, along with co-existing illness and CRB-65 score, raised proANP and proAVP were the strongest predictors of mortality. These biomarkers therefore appear to have potential in the risk stratification of patients with CAP.

A similar degree of predictive value has been demonstrated for another prohormone, namely pro-adrenomedullin (proADM). In a prospective study conducted in $\mathbf{3 0 2}$ patients admitted to the emergency department with CAP [12], proADM levels increased with higher PSI score, and proADM levels on admission were found to have been higher in patients who subsequently died than in survivors.

PCT is the marker that has been most widely investigated and most frequently used in CAP. It has also become the marker of choice for distinguishing between infectious and noninfectious aetiologies. However, several other pro-
Figure 2

\section{MR-proANP}

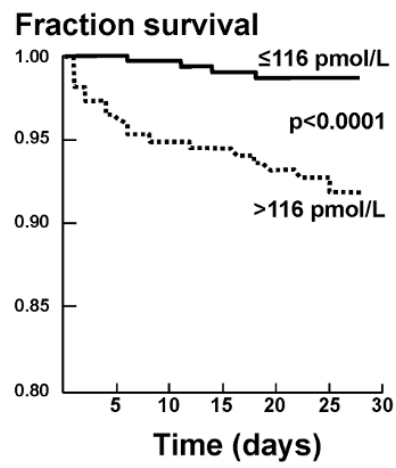

\section{CT-proAVP}

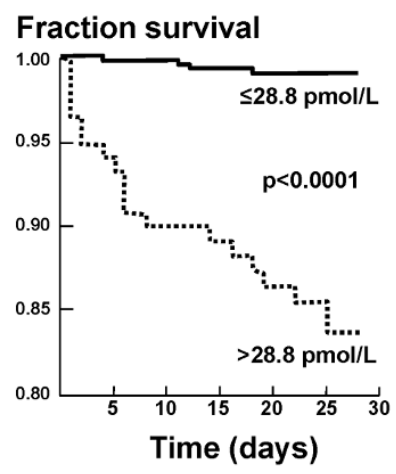

Prognostic value of ProANP and ProAVP in CAP. ProANP and proAVP at levels below $116 \mathrm{pmol} / \mathrm{l}$ and $28.8 \mathrm{pmol} / \mathrm{l}$, respectively, are predictive of survival in CAP patients over a 30-day period. Reproduced with permission from Krüger and coworkers [11]. CAP, community-acquired pneumonia; proANP, pro-atrial natriuretic peptide; proAVP, provasopressin.

hormones appear to have a similar ability to discriminate disease severity.

\section{Markers of inflammation}

The Health Aging and Body Composition study [13] has been prospectively gathering longitudinal data in a cohort of 3,075 initially healthy people aged between 65 and 74 years. Over 6.5 years of follow up, 162 experienced an episode of CAP severe enough to require hospitalization. Risk was greatest among people whose baseline plasma levels of both tumour necrosis factor (TNF)- $\alpha$ and IL- 6 were in the highest tertile (adjusted risk 2.8). Furthermore, people whose marker levels were in the highest third for either TNF- $\alpha$ or IL- 6 had intermediate risk (adjusted risk 1.6); and those whose levels were below the third tertile on both markers were least likely to develop CAP. This pattern was unaltered when people were stratified by potentially confounding factors such as smoking history, reduced lung function, and diabetes or other co-morbid conditions. These two cytokines were previously associated with severity of sepsis. Hence, it is interesting that, among the 1,886 patients initially enrolled in the GenIMS study of CAP, the 583 patients who developed severe sepsis (31\% of the cohort) had a higher death rate than those who did not [14]. At 90 days, the mortality rates were $25 \%$ versus 5\%; and at 1 year they were $35 \%$ versus less than $20 \%$.

The relationship of inflammatory markers in sepsis and its outcome is also interesting [14]. Throughout the first week of hospitalization, the mean level of the proinflammatory cytokine IL-6 was significantly higher in patients who developed severe sepsis and died than in patients who developed sepsis but survived. Mean IL- 6 levels were lowest in patients 
who did not develop sepsis at all. The pattern was similar for the anti-inflammatory cytokine IL-10. However, although levels of this cytokine were clearly high in patients who subsequently died from sepsis, the difference between survivors of sepsis and those not developing the condition was less clear cut.

The close association of these cytokines with poor outcome is also evident in the hazard ratios (HRs) calculated in this study [14]. Among patients in the highest tertile for both IL-6 and IL-10, the HR for death from sepsis was 20.5. In those with medium levels of both markers the HR was around 5, and patients with low levels of both IL-6 and IL-10 at admission were at very low risk for developing severe sepsis and had excellent prospects for survival. The HRs based on high cytokine levels were far higher than the HRs associated with older age (Figure 3). The GenIMS study also provided clear evidence of the prognostic significance of elevated IL-6 and IL-10 measured at the time of hospital discharge [15]. Death rates in the subsequent year reached $20 \%$ for those with high IL-6 levels and 15\% for those with high levels of IL-10. For patients with low levels of these cytokines at discharge, the likelihood of dying was approximately one-third lower.

\section{Markers of coagulation}

Our understanding of the potential prognostic value of coagulation markers is hampered by the paucity of large studies. One study that contributes useful data is that by QuerolRibelles and coworkers [16], who prospectively investigated the relationship between plasma D-dimer level and mortality in 302 consecutive CAP patients admitted to a single hospital. Overall, D-dimer levels were significantly higher in patients with severe CAP who died than in those who survived (mean $3,786 \mathrm{ng} / \mathrm{ml}$ versus $1,609 \mathrm{ng} / \mathrm{ml} ; P<0.0001)$.

Among patients in the PSI V category (those with the most severe disease clinically), the probability of death rose steadily from $8 \%$ when baseline D-dimer levels were below $500 \mathrm{ng} / \mathrm{ml}$ to $77 \%$ when they exceeded $5,000 \mathrm{ng} / \mathrm{ml}$. Among patients in PSI category IV, mortality remained minimal until D-dimer levels exceeded $2,000 \mathrm{ng} / \mathrm{ml}$, at which point the death rate was $10 \%$. Mortality rose to $21 \%$ when D-dimer levels reached $5,000 \mathrm{ng} / \mathrm{ml}$ and greater (Figure 4). Raised Ddimer levels also predicted need for mechanical ventilation, itself a marker for severe CAP.

Other coagulation markers under investigation as having potential utility as biomarkers for indicating the presence of coagulatory disorders associated with severe CAP include prothrombin fragment 1.2, thrombin-antithrombin complexes and fibrinogen.

\section{Genetic markers of susceptibility and prognosis}

Links have been established between many genetic polymorphisms and risk for severe sepsis and CAP. These links
Figure 3

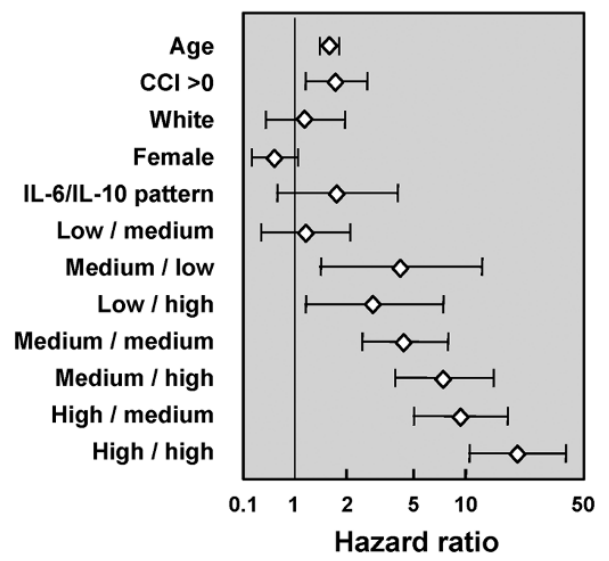

Plasma cytokine concentrations and CAP severity and mortality. Results are from a Cox proportional hazards model for mortality. Points estimates are shown along with their $95 \%$ confidence intervals. Age (increase in age of 10 years), co-morbidity $(\mathrm{CCl})$ and high levels of cytokines (IL-6 or IL-10) are associated with death. The combination of high concentrations of IL- 6 and IL-10 was associated with a 20 times increased risk for death. Reproduced with permission from Kellum and coworkers [14]. CAP, community-acquired pneumonia; CCl, Charlson Comorbidity Index; IL, interleukin.

can be divided into those that affect genes involved in pathogen detection (such as those of Toll-like receptors or CD-14), those influencing inflammatory processes (genes involving the TNF locus or IL-6, IL-10 and IL-18, for example), and those related to coagulation, such as plasminogen activator inhibitor-1, factor $\mathrm{V}$, protein $\mathrm{C}$ and fibrinogen.

Patients with single nucleotide polymorphisms (SNPs) associated with high levels of TNF are at enhanced risk for developing septic shock. Waterer and coworkers [17] investigated 343 patients admitted to a single hospital system with CAP, 30 of whom had septic shock, and determined prospectively the presence or absence of SNPs at the heat shock protein 70-2 and lymphotoxin- $\alpha+250$ loci. Patients homozygous for AA heat shock protein 70-2 had a $20 \%$ chance of developing septic shock, a relative risk of 3.5 compared with patients without an SNP at this site. Presence of the lymphotoxin- $\alpha+250$ AA genotype was associated with a $15 \%$ risk for developing septic shock and a relative risk of 2.7. This study provides clear evidence of the susceptibility conferred by variations in the genes encoding these important immunomodulatory proteins.

In the GenIMS study described above, high levels of IL-10 in CAP patients were linked to increased risk of death from sepsis. Work relevant to the genetics underlying this association was carried out by a Canadian group who studied a cohort of 550 Caucasian patients with sepsis, 158 of whom 
Figure 4

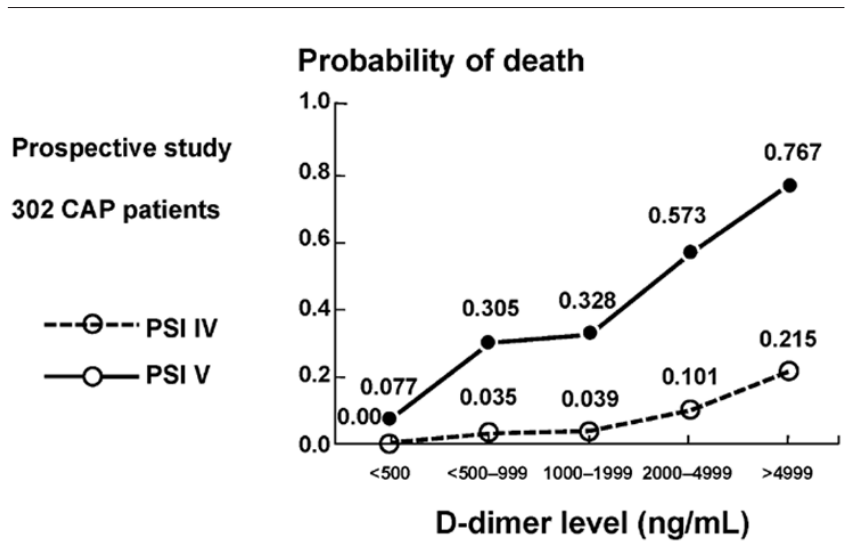

Plasma D-dimer levels correlate with PSI severity and outcomes in CAP patients. The probability of death in relationship with $\mathrm{D}$-dimer levels in PSI categories IV and V is shown. D-dimer levels correlate well with increasing probability of death in PSI IV and V patients. Probabilities were calculated with a logistic regression model using $\mathrm{PSI}$ and D-dimer levels as independent variables. Reproduced with permission from Querol-Ribelles and coworkers [16]. CAP, community-acquired pneumonia; PSI, Pneumonia Severity Index.

had pneumonia as the primary source [18]. By investigating three SNPs in the IL-10 gene, the group identified a haplotype associated with increased mortality in pneumonia patients with sepsis. Those with one or two copies of the haplotype had a 28 -day mortality of $51 \%$, as compared with $29 \%$ in noncarriers. Carriers of the haplotype also required greater use of vasopressors and had greater renal, hepatic and haematological dysfunction than did noncarriers. Interestingly, the risk conferred by the IL-10 haplotype was confined to patients with sepsis related to pneumonia. Presence of the SNPs did not predict higher risk for mortality or organ dysfunction in patients with sepsis of similar severity deriving from nonpulmonary sites of infection.

Polymorphisms related to the coagulation system have also been linked to susceptibility to development of pneumonia. Thus, workers in Pittsburgh [19] established that the 4G/5G polymorphism and other mutations that increase plasminogen activator inhibitor-1 expression doubled the likelihood that elderly white people enrolled in the Health $A B C$ cohort would experience an episode of CAP during a 12-year follow-up period.

Such a finding does not define specific strategies for the primary prevention of CAP, but it contributes to our confidence that genetic factors that are useful in managing CAP will be identified in years to come. With increasing knowledge of the inherited factors that underlie expression of molecules relevant to outcome in CAP, in the future it should become possible to tailor the management of an individual patient to his or her specific genotype [20].
Figure 5

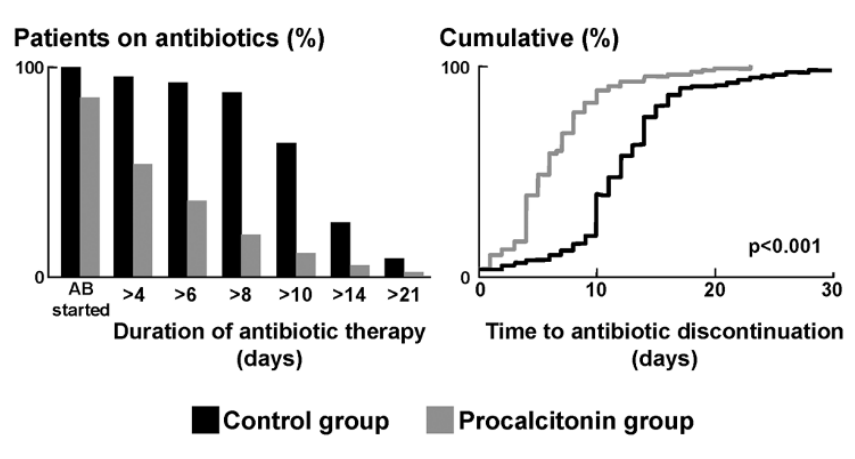

PCT-guided antibiotic therapy in CAP leads to reduced time on antibiotics and antibiotic use. (a) Percentage of patients receiving antibiotic therapy in the control group and the procalcitonin group on admission and during the course of the disease. (b) Cumulative frequency distribution curve for the time to discontinuation in patients for whom antibiotic therapy was prescribed. Patients in the procalcitonin group were compared with those in the control group. Reproduced with permission from Christ-Crain and coworkers [21]. $A B$, antibiotics.

\section{Biomarkers and treatment decisions}

Christ-Crain and colleagues [21] in Basel have pioneered the strategy of using PCT to guide the management of CAP in patients arriving at the emergency department. In a recent study, 404 patients were randomized at entry to antibiotic treatment guided by PCT or to a group in which antibiotics were given according to standard protocols. The group in which antibiotics were given or withheld according to the PCT level were less likely to receive the drugs (80\% versus $100 \%$ ) and had a shorter duration of antibiotic therapy (5 days versus $>12$ days). Moreover, use of antibiotics was more attuned to disease severity; PCT-guided treatment resulted in patients in the low-risk PSI groups (I to III) being treated for 4 days, whereas the standard protocol approach led them to be treated for 12 days (as long as patients in higher severity groups; Figure 5).

Most importantly, the intent-to-treat analysis revealed that guiding antibiotic use by PCT was associated with the same clinical outcome as standard protocol, which generated far greater use of antibiotics. Confirmation of this finding is now being sought in a prospective randomized controlled trial in more than 1,000 patients with lower respiratory tract infections [22]; demonstrating noninferiority of the PCT-guided approach will be the primary outcome.

Evidence of the potential benefit of PCT was also found in a study conducted by Menendez and coworkers [23], which suggests that the marker can be helpful in identifying the $10 \%$ to $15 \%$ of CAP patients in whom treatment will fail (a situation associated with mortality of about $40 \%$ ). Menendez and coworkers prospectively measured CRP, PCT and 
cytokines at days 1 and 3 , looking for a relationship with treatment failure defined by need of mechanical ventilation, vasopressors or other forms of organ support. IL-6 level at day 1 was significantly related to late treatment failure (failure occurring after 72 hours). However, PCT was predictive of early failure (within the first 72 hours), with a sensitivity of $57 \%$ and a specificity of $75 \%$ [23]. It may therefore have particular value in identifying patients at high risk for disease progression.

Subgroup analysis of the placebo-controlled OPTIMIST trial of tifacogin in severe sepsis revealed a trend toward benefit in patients with procalcitonin levels of $2 \mathrm{ng} / \mathrm{ml}$ or greater and in those with high baseline markers of activated coagulation (unpublished data). Changes in biomarkers during the course of disease may enable us to identify those patients who are most at risk for deterioration and in greatest need of early intervention. Such studies are being undertaken as part of the ongoing CAPTIVATE study of recombinant tissue factor pathway inhibitor (tifacogin) in severe CAP.

\section{Conclusion}

The management of severe CAP would be greatly improved if it were possible to identify, early in the course of disease, those patients who are most likely to develop severe complications. However, at the moment, the greatest utility of clinical scoring systems appears to lie in detecting patients at low risk. Several biomarkers are good options for identifying patients who are likely to develop more severe CAP. Although the full potential of PCT must still be established, it appears that it may be a helpful marker of high risk, as well as having the valuable role of distinguishing between infectious and noninfectious aetiologies of organ dysfunction.

There are other prohormones that relate to disease severity, and the proinflammatory cytokine IL- 6 and the anti-inflammatory cytokine IL-10 are also clearly involved. Cytokines are prognostic for development of CAP in healthy people, for development of sepsis and death in hospitalized patients, and for mortality after hospital discharge after treatment for pneumonia and sepsis.

In considering the range of possible markers, it may be useful to distinguish between those that reflect processes that are clearly pathological and those that reflect aspects of the host response to infection. None of these markers should ever be used in isolation to make clinical decisions, and it is clear that the role of laboratory data to help identify patients who are likely to experience a poor outcome remains to be defined. Nevertheless, it would appear that patients at risk, and so eligible for trials of novel approaches, are those with PSI IV or $\mathrm{V}$ disease with elevated PCT and/or D-dimer.

In the near future there may be clinically useful tools for identifying patients with high-risk cytokine profiles and the genotypes that underlie them. Such techniques would be useful for deciding on both the intensity of care required for a particular patient and the appropriateness of specific interventions. We might hypothesize, for example, that mutations in genes that are relevant to coagulation and inflammation, and hence to the mechanisms of action of potential adjunctive therapies, will influence the degree of benefit derived from its use. It is also plausible that differences in the pathophysiology of sepsis arising at different sites might explain why some adjunctive therapies could be effective in patients with lung injury while not improving outcome in others.

Severe CAP may be considered as a systemic response to an initially local infection. The invading pathogen stimulates an inflammatory cytokine release, and disrupts the balance of procoagulant and anticoagulant factors - responses that can both be measured in the laboratory through the use of biomarkers. An 'ideal' panel of tests for determining a positive diagnosis of severe CAP perhaps should reflect the three components of the systemic response: infection, inflammation and haemostasis. Current clinical practice involves determining the presence of infection, usually through cultures, Gram stain, urine antigen testing or serology, but it does not involve routine assessment of the patient's level of inflammatory response or for disorders of coagulation. Perhaps the routine inclusion of additional tests for patients with suspected severe CAP such as PCT and coagulation markers (for example, D-dimer, prothrombin fragment 1.2 or thrombinantithrombin complexes) may identify patients who require additional supportive care to address these disorders, either through admission to ICU or through initiation of additional treatment.

\section{Competing interests}

In the last five years, JPM has received fees from Novartis, Eli Lilly, LFB and MSD. AM and PRB declare that they have no competing interests.

\section{Acknowledgement}

This article is based on a presentation made at a satellite symposium, 'Severe community-acquired pneumonia update: mortality, mechanisms and medical intervention', held on 21 April 2008 in Barcelona, Spain as part of the 18th European Congress of Clinical Microbiology and Infectious Diseases (ECCMID). It is published as part of Critical Care Volume 12 Supplement 6, 2008. The full contents of the supplement are available online at http://ccforum.com/supplements/12/S6

Publication of the supplement has been sponsored by Novartis.

\section{References}

1. Niederman MS, McCombs JS, Unger AN, Kumar A, Popovian R: The cost of treating community-acquired pneumonia. Clin Ther 1998, 20:820-837.

2. MacFarlane JT, Boldy D: 2004 update of BTS pneumonia guidelines: what's new? Thorax 2004, 59:364-366.

3. Restrepo Ml, Anzueto Z: The role of new therapies for severe community-acquired pneumonia. Curr Opin Infect Dis 2006, 19:557-564.

4. Kaplan V, Clermont G, Griffin MF, Kasal J, Watson RS, LindeZwirble WT, Angus DC: Pneumonia: still the old man's friend? Arch Intern Med 2003, 163:317-323.

5. Rello J: Demographics, guidelines and clinical experience in 
severe community-acquired pneumonia. Crit Care 2008, 12 (Suppl 5):S2.

6. Mandell LA, Wunderlink RG, Anzueto A, Bartlett JG, Campbell D, Dean NC, Dowell SF, File TM, Musher DM, Niederman MS, Torres A, Whitney CG: Infectious Diseases Society of America/American Thoracic Society consenses guidelines on the management of community-acquired pneumonia in adults. Clin Infect Dis 2007, 44:S27-S72.

7. Barlow G, Nathwani D, Davey P: The CURB65 pneumonia severity score outperforms generic sepsis and early warning scores in predicting mortality in community-acquired pneumonia. Thorax 2007, 62:253-259.

8. Man YS, Lee N, Ip M, Antonio GE, Chau SS, Mak P, Graham GA, Zhang M, Lui G, Chan PKS, Ahuja AT, Hui DS, Sung JJ, Rainer $\mathrm{TH}$ : Prospective comparison of three predictive rules for assessing severity of community acquired pneumonia in Hong Kong. Thorax 2007, 62:348-353.

9. Muller B, Harbarth S, Stolz D, Bingisser R, Muller C, Leuppi J, Nusbaumer C, Tamm M, Christ-Crain M: Diagnostic and prognostic accuracy of clinical and laboratory parameters in community-acquired pneumonia. BMC Infect Dis 2007, 7:10

10. Huang D, Weissfeld LA, Kellum JA, Yealy DM, Kong L, Martino M, Angus DC; GenIMS Investigators: Risk prediction with procalcitonin and clinical rules in community-acquired pneumonia. Ann Emerg Med 2008, 52:48-58.

11. Kruger S, Papassotiriou J, Marre R, Richter K, Schumann C, von Baum H, Morgenthaler NG, Suttorp N, Welte T; CAPNETZ Study Group: Pro-atrial natriuretic peptide and pro-vasopressin to predict severity and prognosis in community acquired pneumonia: results from the German competence network CAPNETZ. Intensive Care Med 2007, 33:2069-2078.

12. Christ-Crain M, Morgenthaler NG, Stolz D, Muller C, Bingisser R, Harbarth S, Tamm M, Struck J, Bergmann A, Muller B: Proadrenomedullin to predict severity and outcome in community acquired pneumonia. Crit Care 2006, 10:R96.

13. Yende $S$, Tuomanen El, Wunderink R, Kanaya A, Newman AB, Harris T, de Rekeneire N, Kritchevsky SB: Preinfection systemic inflammatory markers and risk of hospitalization due to pneumonia. Am J Resp Crit Care Med 2005, 172:1440-1446.

14. Kellum J, Kong L, Fink MP, Weissfeld LA, Yealy DM, Pinsky MR, Fine J, Krichevsky A, Delude RL, Angus DC; GenIMS investigators: Understanding the inflammatory cytokine response in pneumonia and sepsis: results of the Genetic and Inflammatory Markers of Sepsis (GenIMS) study. Arch Intern Med 2007, 167:1655-1663.

15. Yende S, D'Angelo G, Kellum JA, Weissfeld L, Fine J, Welch RD, Kong L, Carter M, Angus DC; GenIMS Investigators: Inflammatory markers at hospital discharge predict subsequent mortality after pneumonia and sepsis. Am J Respir Crit Care Med 2008, 177:1242-1247.

16. Querol-Ribelles JM, Tenias JM, Grau E, Querol-Borras JM, Climent JL, Gomez E, Martinez I: Plasma d-dimer levels correlate with outcomes in patients with community-acquired pneumonia. Chest 2004, 126:1087-1092.

17. Waterer GW, ElBahlawan L, Quasney MW, Zhang Q, Kessler LA, Wunderink RG: Heat shock protein 70-2+1267 AA homozygotes have an increased risk of septic shock in adults with community-acquired pneumonia. Crit Care Med 2003, 31: 1367-1372.

18. Wattanathum A, Manocha S, Groshaus $H$, Russell JA, Walley KR: Interleukin-10 haplotype associated with increased mortality in critically ill patients with sepsis from pneumonia but not in patients with extrapulmonary sepsis. Chest 2005, 128:16901698.

19. Yende S, Angus DC, Ding J, Newman AB, Kellum JA, Li R, Ferrell RE, Zmuda J, Kritchevsky SB, Harris TB, Garcia M, Yaffe K, Wunderink RG; for the Health $A B C$ Study: 4G/5G plasminogen inhibitor-1 polymorphisms and haplotypes are associated with pneumonia. Am J Resp Crit Care Med 2007, 176:11291137.

20. Wunderink RG, Waterer GW: Genetics of community-acquired pneumonia. Semin Resp Crit Care Med 2005, 26:553-562.

21. Christ-Crain $M$, Stolz D, Bingisser R, Muller C, Miedinger D, Huber PR, Zimmerli W, Harbarth S, Tamm M, Muller B: Procalcitonin guidance of antibioitic therapy in community-acquired pneumonia: a randomized trial. Am J Resp Crit Care Med 2006, 174:84-93.
22. Schuetz P, Christ-Crain M, Wolbers M, Schild U, Thomann R, Falconnier C, Widmer I, Neidert S, Blum CA, Schoneberger R, Henzen C, Bregenzer T, Hoess C, Krause M, Bucher HC, Zimmerli W, Muller B; ProHOSP study group: Procalcitonin guided antibiotic therapy and hospitalization in patients with lower respiratory tract infections: a prospective, multicenter, randomized controlled trial. BMC Health Serv Res 2007, 7:102.

23. Menendez R, Cavalcanti M, Reyes S, Mensa J, Martinez R, Marcos MA, Filella X, Niederman M, Torres A: Markers of the treatment failure in hospitalized community acquired pneumonia. Thorax 2008, 63:447. 